\title{
Ausdauertraining bei gleichzeitiger Kälteadaptation: Auswirkungen auf den Muskelstoffwechsel
}

\author{
Angela Schuh \\ Institut für Medizinische Balneologie und Klimatologie der Ludwig-Maximilians-Universität München \\ (Vorstand: Prof. Dr. med. E. Senn)
}

\section{Endurance training under simultaneous cold adaption: effects on muscle metabolism}

Objective: In the past years several studies documented an immediate reaction of heart rate under the influence of cold. But there has yet no research been done about immediate reaction on aerobic muscle metabolism under these conditions. About the connection between the process of a developing state of endurance training under simultaneous exposure to cold, in the sense of a cross adaption or an additive effect, no relevant studies can be found in literature, too. So far no author could point out, whether the adaptation to a cool environment during endurance training, goes along with an increased efficacy.

Subjects: The goal of the here presented study was therefore to quantify the improvement of aerobic performance by endurance training with and without cooling of body shell, to prove a cross adaptation. At the same time was to be observed, whether cool conditions do not only improve endurance training effect, but whether an immediate effect on aerobic performance could be proved. Above that, that study should answer the question, whether these effects occur, when the training subject's body core is cooled, or whether reducting the temperature of body shell would be enough. The effectiveness of isolated cooling of the body shell during training had to be proved.

Design: During endurance training with simultaneous cold adaptation the effects of a cool body shell on the muscle metabolism was examined. To do this terrain cures, lasting three weeks, were conducted: With one group of test subjects ( $\mathrm{n}=63$ ) the body shell was kept defined cool during a terrain walk; the skin temperature was lowered by $2 \mathrm{C}$, the core temperature was not influenced. The control group $(n=61)$ did a terrain cure under thermally balanced conditions. A third group $(\mathrm{n}=29)$ did not participate in the therapy. The effect of endurance training was evaluated using ergometric tests, taken at the begin and at the end of the therapy. Additionally the actual aerobic fitness was measured under different thermal conditions. Most important measuring parameter was the blood lactate concentration.

Results: The result showed, that reduced skin temperature, during work, has two effects on the muscle metabolism:

- As immediate effect, the actual aerobic capacity of muscle metabolism is increased: Doing the same work the

\section{Kurzfassung}

Problemstellung: In den letzten Jahren wurden durch mehrere Studien Sofortreaktionen der Herzfrequenz während Kälteeinwirkung auf die Haut dokumentiert. Sofortreaktionen auf den aeroben Muskelstoffwechsel unter diesen Bedingungen wurden dagegen nicht untersucht. Über die Zusammenhänge zwischen dem Prozeß eines sich entwickelnden Ausdauertrainingszustandes bei gleichzeitiger Kälteexposition im Sinne einer Kreuzadaptation oder eines additiven Effektes finden sich in der Literatur ebenfalls keine relevanten Studien. Bisher konnte von keinem Autor dargelegt werden, ob die Adaptation an kühle Umgebungsbedingungen während eines Ausdauertrainings mit einer erhöhten Wirksamkeit desselben einhergeht.

Gegenstand: Das Ziel der vorliegenden Studie war es, die Verbesserung der aeroben Leistungsfähigkeit durch ein Ausdauertraining mit und ohne Abkühlung der Körperschale zu quantifizieren, um eine allfällige Kreuzadaptation zu belegen. Gleichzeitig sollte festgelegt werden, ob sich durch die kühlen Bedingungen nicht nur eine Verbesserung des Ausdauertrainingseffektes, sondern auch ein Soforteffekt auf die aerobe Leistungsfähigkeit nachweisen läßt. Darüber hinaus sollte die Frage beantwortet werden, ob diese Effekte erst bei einer Abkühlung des Trainierenden bis in den Körperkern auftreten oder ob bereits eine Reduzierung der Temperatur der Körperschale ausreicht: Die Wirksamkeit der isolierten Abkühlung der Körperschale während des Trainings sollte bewiesen werden.

Versuchsplan: Während eines Ausdauertrainings mit gleichzeitiger Kälteadaptation (3wöchige klimatische Terrainkur) wurden die Auswirkungen einer kühlen Körperschale auf den Muskelstoffwechsel untersucht: Bei einer Probandengruppe ( $n=63$ ) wurde die Körperschale während der Terrainwanderung definiert kühl gehalten; die Hauttemperatur wurde um $2{ }^{\circ} \mathrm{C}$ erniedrigt, die Kerntemperatur nicht beeinflußt. Die Kontrollgruppe $(n=61)$ absolvierte eine Terrainkur unter thermisch ausgeglichenen Bedingungen. Eine dritte Probandengruppe ( $n=29)$ nahm am Therapieprogramm nicht teil. Der Ausdauertrainingseffekt wurde mittels ergometrischer Tests, welche am Therapieanfang und Therapieende vorgenommen wurden, untersucht. Zusätzlich wurde dabei die aktuelle aerobe Leistungsfähigkeit unter verschiedenen thermischen Bedingungen festgestellt. Wichtigster Meßparameter war der Milchsäurespiegel 
lactate concentration is under cool conditions by $0,4 \mathrm{mmol} / \mathrm{l} \mathrm{sig-}$ nificantly lower $(\mathrm{p} \leq 0.01)$.

- In the long term, the training effect is almost doubled by the cold induced improvement of aerobic metabolism: The subjects, who trained under lowered skin temperature, showed a by $1 \mathrm{mmol} / \mathrm{l}$ significantly ( $\mathrm{p} \leq 0.01$ ) higher training effect than the control group.

Conclusions: The increase of the muscle metabolism's aerobic capacity, because of the cool body shell, makes the actual aerobic mastering if work easier and doubles the endurance training effect. Therefore, these results have clinical relevance for patients with low exercise tolerance: Adaptations are made possible at relatively low training intensity.

\section{Key words}

Muscle metabolism - endurance training - cold adaptation
Ergebnisse: Eine reduzierte Hauttemperatur während Arbeit hat zwei Auswirkungen auf den Muskelstoffwechsel:

- Als Soforteffekt wird die aktuelle aerobe Kapazität des Muskelstoffwechsels vergrößert: Bei gleicher Arbeit liegt der Laktatspiegel unter kühlen Bedingungen um $0,4 \mathrm{mmol} / \mathrm{l}$ signifikant $(\mathrm{p} \leq 0,01)$ niedriger.

- Langfristig wird der Trainingseffekt durch die kältebedingte Verbesserung des aeroben Metabolismus annähernd verdoppelt: Die Personen, welche bei erniedrigter Hauttemperatur trainierten, wiesen einen um knapp $1 \mathrm{mmol} / \mathrm{l}$ signifikant ( $p \leq 0,01$ ) größeren Trainingseffekt als die Kontrollgruppe auf.

Schlußfolgerung: Die Vergrößerung der aeroben Kapazität des Muskelstoffwechsels aufgrund der kühlen Körperschale erleichtert die aktuelle aerobe Bewältigung der Arbeit und verdoppelt den Ausdauertrainingseffekt. Damit haben diese Ergebnisse eine klinische Relevanz für Patienten, welche nur leicht belastbar sind: Adaptationen werden schon bei relativ geringer Trainingsintensität ermöglicht.

\section{Schlüsselwörter}

Muskelstoffwechsel - Ausdauertraining - Kälteadaptation

\section{Einleitung}

Bei Patienten mit Funktionsstörungen des Herzkreislaufsystems ohne Organbefund kann eine unterdurchschnittlich kardiopulmonale Leistungsfähigkeit und eine starke Häufung von Allgemeinsymptomen bzw. Störungen der Befindlichkeit festgestellt werden (25). Es konnte gezeigt werden (8), daß diesen Erkrankungen im Rahmen multikausaler Faktoren ein Trainingsmangel des ganzen Körpers zugrunde liegt. Dieser Trainingsmangel beinhaltet über sportmedizinische Aspekte hinaus, aufgrund der fehlenden Auseinandersetzung mit ihnen, auch die mangelhafte Reaktion auf thermische Reize.

Zur Therapie der nichtorganischen Funktionsstörungen des Herz-Kreislauf-Systems muß folgerichtig sowohl ein Training auf Ausdauer als auch ein spezielles Thermoregulationstraining durch Kälteadaptation durchgeführt werden. Die Umsetzung dieser Kombination stellt eine Terrainkur (d. h. kurmäßig dosiertes Gehen auf ansteigenden Wegen) unter kühlen Bedingungen (22) dar.

Die Auswirkungen kühler Körpertemperaturen auf das Verhalten physiologischer Parameter einschließlich der maximalen Intensität einer aktuellen Arbeitsleistung sind weitgehend bekannt $(2,4,11,13,15,16,26)$. In den letzten Jahren wurden durch mehrere Studien Sofortreaktionen der Herzfrequenz während Kälteeinwirkung auf die Haut dokumentiert: Bei Fahrradergometerarbeit unter kühlen Bedingungen - mit Kältereizen auf die gesamte Körperoberfläche - ist eine gegenüber einer Arbeitsleistung bei Raumtemperatur verminderte Herzfrequenz als gesichert anzusehen (26). Gleichzeitig sind Sauerstoffaufnahme und Schlagvolumen vergrößert (13). Wenn während Fahrradergometerarbeit nur das Gesicht mit kalter Luft angeblasen wird, zeigt sich eben- falls eine Senkung der Herzfrequenz (11). Ein größerer Effekt ergibt sich dann, wenn nicht nur die Hauttemperatur, sondern gleichzeitig auch die Kerntemperatur gesenkt wird. Wegweisend sind dabei die Studien der Arbeitsgruppe um Brück (2, 4, 15, 16): Bei Fahrradergometerbelastung nach Vorkühlung, wobei die Körpertemperatur als Mittel von Kern- und mittlerer Hauttemperatur um 1 ${ }^{\circ} \mathrm{C}$ gesenkt wird, sind bei gegebener Herzfrequenz Arbeitsleistung und $\mathrm{O}_{2}$-Puls signifikant höher; die Ausdauerleistungsfähigkeit ist vergrößert. Sofortreaktionen des aeroben Muskelstoffwechsels unter diesen Bedingungen wurden bisher nicht untersucht.

Über die Zusammenhänge zwischen dem Prozeß eines sich entwickelnden Ausdauertrainingszustandes bei einer gleichzeitigen Kälteexposition im Sinne einer Kreuzadaptation oder eines additiven Effektes finden sich in der Literatur keine relevanten Studien. Bisher konnte von keinem Autor dargelegt werden, ob die Adaptation an kühle Umgebungsbedingungen während eines Ausdauertrainings mit einer erhöhten Wirksamkeit desselben einhergeht.

In ersten eigenen Studien (23) fanden sich bei Patienten mit Funktionsstörungen des Herz-Kreislauf-Systems ohne Organbefund, die während einer Terrainkur im Winter trainiert wurden, anhand des Laktatspiegels erste Hinweise auf eine Unterstützung des Ausdauertrainingseffektes durch die kühlen Umgebungsbedingungen während des Trainings.

Das Ziel der vorliegenden Studie war es deshalb, die Verbesserung der aeroben Leistungsfähigkeit durch ein Ausdauertraining mit und ohne Abkühlung der Körperschale zu quantifizieren, um eine allfällige Kreuzadaptation zu belegen. 
Gleichzeitig sollte festgestellt werden, ob sich durch die kühlen Bedingungen nicht nur eine Verbesserung des Ausdauertrainingseffektes, sondern auch ein Soforteffekt auf die aerobe Leistungsfähigkeit nachweisen läßt.

Darüber hinaus war die Frage zu beantworten, ob diese Effekte erst bei einer Abkühlung des Trainierenden bis in den Körperkern auftreten oder ob bereits eine Reduzierung der Temperatur der Körperschale ausreicht. Die Wirksamkeit der isolierten Abkühlung der Körperschale während des Trainings sollte bewiesen werden.

\section{Methodik}

An den Studien zur Überprüfung der Auswirkungen einer Kombination von Ausdauertraining und gleichzeitiger Kälteadaptation auf die Milchsäureproduktion haben insgesamt 153 Personen, 82 Frauen und 71 Männer, mit einem durchschnittlichen Alter von 41 Jahren teilgenommen. Der jüngste Proband war 16, der älteste 72 Jahre. Davon waren 83 unterdurchschnittlich trainierte Kurpatienten mit Funktionsstörungen des Herz-Kreislauf-Systems ohne Organbefund; sie wurden aufgrund von Befindlichkeitsstörungen, die zu den entsprechenden kurärztlichen Diagnosen (Tab. 1) führten, in die Studie aufgenommen.

Die restlichen 90 Kurpatienten waren herz-kreislaufgesund; ihr Trainingszustand entsprach dem der Normalbevölkerung (Tab. 2). Alle Probanden wurden randomisiert in drei Gruppen aufgeteilt (Tab. 3): Die „Kühlgruppe“ $(n=63)$ führte eine klimatische Terrainkur bei gleichzeitiger Körperschale durch; die „Kontrollgruppe“ ( $n=61)$ absolvierte eine konventionelle Terrainkur unter thermisch ausgeglichenen Bedingungen. Die Personen der "Nullgruppe“ ( $n=29)$ nahmen am Therapieprogramm nicht teil.

Die jeweils dreiwöchigen klimatischen Terrainkuren wurden in Garmisch-Partenkirchen (800-1800 m ü. NN) und Davos (1600-2000 m ü. d. Meer) durchgeführt.

Tab. 1 Einschlußkriterien für Patienten mit Funktionsstörungen des Herz-Kreislauf-Systems ohne organische Ursache. Einzeldiagnosen der kurärztlichen Untersuchungen, Mehrfachnennungen möglich ( $n$ $=167$ )

\begin{tabular}{lr} 
hypotone Funktionsstörungen & $49,7 \%$ \\
essentielle hypertone Funktionsstörungen & $21,0 \%$ \\
Herzrhythmusstörungen & $19,2 \%$ \\
vagovasale Synkopen, Schwindel & $8,4 \%$ \\
hyperkinetisches Herzsyndrom & $1,2 \%$ \\
Hyperventilationssyndrom & $0,6 \%$ \\
\hline
\end{tabular}

Nach der anfänglichen Überprüfung der Leistungsfähigkeit auf dem Fahrradergometer, erfolgte die Dosierung der Ausdauerleistung (W/kg) über die Gehgeschwindigkeit. Sie wurde je nach Trainingszustand der Patienten mit Hilfe eines Metronoms vorgegeben und reichte von 60 bis 110 Schritten/Minute. Die Dauer der einzelnen Terrainbegehungen wurde von anfänglich 20 Minuten auf maximal 1,5 Stunden gesteigert. Das Therapieprogramm wurde so gestaltet, daß jeder Patient ohne Rücksicht auf die Wetterlage während seiner Kur 4mal pro Woche trainierte.

Die Wanderungen erfolgten für Kühl- und Kontrollgruppe gemeinsam, d. h. zur selben Tageszeit, bei gleichen Wetterbedingungen, auf denselben Wegen und mit gleicher Geschwindigkeit. Die Anpassung der Gehgeschwindigkeit an die aktuelle mittlere körperliche Leistungsfähigkeit des Patientenkollektivs wurde durch Herzfrequenzmessungen, die bei jeder einzelnen Begehung an festgelegten Ortspunkten erfolgten, vorgenommen. Maßgabe war der Trainingspuls (130/Minute) der Patienten der Kontrollgruppe: Aufgrund der unterschiedlichen thermischen Bedingungen während des Trainings wanderten somit die Patienten der Kühlgruppe bei gleicher Leistung wir die Kontrollgruppe stets mit etwas niedrigerer Herzfrequenz (kältebedingt reduzierte Arbeitstachykardie).

Die thermischen Bedingungen waren für die zwei Therapiegruppen unterschiedlich:

Bei den Patienten der Kühlgruppe sollte die Körperschale während der einzelnen Terrainwanderungen definiert abgekühlt bzw. kühl gehalten werden. Dies wurde durch Vorgabe der Bekleidung gewährleistet, basierend auf bekannten Zusammenhängen zwischen der Wärmebilanz während körperlicher Arbeit, dem subjektiven thermischen Empfinden und dazugehörenden Bekleidungsisolationswerten in Abhängigkeit von den klimatischen Umgebungsbedingungen (21).

Die Hauttemperatur wurde mit Thermoelementen, die während der Begehungen an definierten Körperstellen fixiert waren, gemessen. Die mittlere Hauttemperatur wurde aus den

Tab. 3 Einteilung aller Patienten in drei Gruppen: Trainingszustand gemessen anhand Herzfrequenz und Laktat bei Fahrradergometrie (individuell höchste Belastungsstufe) am Kuranfang

\begin{tabular}{llll}
\hline & Kühlgruppe & Kontrollgruppe & Nullgruppe \\
\hline $\mathrm{n}$ & 63 & 61 & 29 \\
& (32 Frauen & (33, Frauen, & (17 Frauen, \\
& 31 Männer) & 28 Männer) & 12 Männer) \\
$\mathrm{HF}\left(\mathrm{min}^{-1}\right)$ & $144( \pm 3)$ & $144( \pm 4)$ & $149( \pm 4)$ \\
Laktat $(\mathrm{mmol} / \mathrm{l})$ & $3,95( \pm 0,3)$ & $4,00( \pm 0,2)$ & $4,3( \pm 0,2)$ \\
\hline
\end{tabular}

Tab. 2 Trainingszustand von Patienten mit Funktionsstörungen des Herz-Kreislauf-Systems ohne Organbefund (FHKE) und herz-kreislaufgesunden Kurpatienten bei 100 W Ergometerbelastung; zusätzlich Werte der Normalbevölkerung. Mittelwerte und Standardabweichung bzw. Referenzbereich

\begin{tabular}{|c|c|c|c|c|}
\hline & \multicolumn{2}{|l|}{ FHKE } & $\begin{array}{l}\text { Herz-Kreislauf- } \\
\text { gesunde Patienten } \\
\bar{x} \quad \text { SEM }\end{array}$ & $\begin{array}{l}\text { Normal- } \\
\text { bevölkerung* } \\
\text { Referenzbereich }\end{array}$ \\
\hline $\begin{array}{l}\text { HF }\left(\mathrm{min}^{-1}\right) \\
R R_{\mathrm{S}}(\mathrm{mmHG}) \\
\mathrm{RR}_{\mathrm{S}} \times \mathrm{HF}\left(\mathrm{mmHgmin}{ }^{-1}\right) \\
\text { Laktat }(\mathrm{mmol} / \mathrm{l})\end{array}$ & $\begin{array}{r}144 \\
200 \\
28400 \\
4,6\end{array}$ & $\begin{array}{l} \pm \quad 4 \\
\pm \quad 5 \\
\pm 1000 \\
\pm 0,3\end{array}$ & $\begin{aligned} 135 & \pm 3 \\
162 & \pm 2 \\
21900 & \pm 300 \\
3,7 & \pm 0,3\end{aligned}$ & $\begin{aligned} & 110- 140 \\
& 150- 170 \\
& 20000-22000 \\
& 2,7-3,7\end{aligned}$ \\
\hline
\end{tabular}

*nach Löllgen et al. 1983 
Temperaturen am Rücken, rechten Oberarm, rechten Oberschenkel und rechten Unterschenkel errechnet (17). Sie wurde in Ruhe, dreimal während einer etwa 20minütigen Begehung und am Ende des Weges bestimmt. Die Kerntemperatur wurde rektal am Anfang und Ende des Terrainweges erhoben. Diese Messung der Hautund Kerntemperaturen beschränkte sich auf 32 Personen der Kontrollgruppe, die zusätzlich zum Trainingsprogramm randomisiert Begehungen sowohl unter thermisch neutralen als auch kühlen Bedingungen durchführten.

Für die Erfolgsbeurteilung wurde die Ausdauerleistungsfähigkeit anhand von zwei Testverfahren erfaßt: Erstens durch eine Fahrradergometrie ( $25 \mathrm{~W}$-Stufen, 3 Minuten, max. 150 W) und zweitens durch standardisierte Gehtests im Terrain $(330 \mathrm{~m}$, $15 \%$ Steigung, 80 Schritte/Minute). Diese Anfangs- und Enduntersuchungen wurden jeweils unter thermisch neutralen Bedingungen durchgeführt.

Zusätzlich wurde am Anfang und Ende der Kur bei einem Teil der Patienten ( $n=42$, zu gleichen Teilen aus Kühlund Kontrollgruppe) die Ausdauerleistungsfähigkeit im Gelände unter kühlen Bedingungen getestet, um den Soforteffekt zu erfassen.

Wichtigster Meßparameter bei der Überprüfung der Ausdauerleistungsfähigkeit war der Laktatspiegel. Er wurde aus dem arterialisierten Ohrläppchenblut, welches in Ruhe, nach 100 Watt (stufenweise Fahrradergometer) und 60 Sekunden nach Belastungsende abgenommen wurde, ermittelt. Die Bestimmung wurde quantitativ mit einem Laktat-Analysator (Roche, Modell 640 ) vorgenommen.

Zur statistischen Sicherung der Daten für paarige und unverbundene Stichproben diente der allgemeine Student-tTest. Für die Irrtumswahrscheinlichkeit wurden folgende Signifikanzniveaus angewendet:

$$
\begin{array}{lll}
\mathrm{p} \leq 0,05 * & * & \text { leicht signifikant } \\
\mathrm{p} \leq 0,01 & * * & \text { signifikant } \\
\mathrm{p} \leq 0,001 * * * & \text { hochsignifikant }
\end{array}
$$

\section{Ergebnisse}

\section{Körpertemperatur}

Die mittlere Hauttemperatur liegt bei jeder der vier während der Begehungen durchgeführten Messungen unter kühlen Bedingungen hochsignifikant $(p \leq 0,001)$ niedriger als im neutralen thermischen Zustand (Abb. 1): Die Kühlgruppe wies im Mittel eine um knapp $2{ }^{\circ} \mathrm{C}$ niedrigere Hauttemperatur auf, als bei Arbeit unter ausgeglichenen thermischen Verhältnissen (Kontrolle). Die Kerntemperatur blieb dabei unbeeinflußt: Während der Begehung steigt die Rektaltemperatur infolge der körperlichen Arbeit sowohl unter thermisch ausgeglichenen als auch unter kühlen Bedingungen leicht an; es besteht keine Abhängigkeit von den thermischen Einflüssen (n. s.).

\section{Aktuelle körperliche Leistungsfähigkeit}

Es ergibt sich unter Arbeitsleistung bei erniedrigter Hauttemperatur eine Verbesserung der aktuellen aeroben Leistungsfähigkeit. Im Akutversuch im Gelände liegt der Laktatspiegel bei gleicher Leistung unter kühlen Bedingungen leicht signifikant $(\mathrm{p} \leq 0,05)$ niedriger als unter thermisch neutralen Verhältnissen (Abb. 2). Dieser Effekt zeigt sich unabhängig von den Auswirkungen des Trainings sowohl bei den Tests am Kuranfang als auch am Kurende. Anfangs liegen die Belastungswerte unter neutralen Bedingungen bei $3,9 \mathrm{mmol} / \mathrm{l}$, im kühlen Zustand bei $3,5 \mathrm{mmol} / \mathrm{l}$. Am Kurende steigt der Laktatspiegel unter gleicher Belastung nur noch auf 3,4 mmol/l (neutral) bzw. auf knapp 3,1 mmol/l (kühl). Der Unterschied von $0,4 \mathrm{mmol} / \mathrm{h}$ bzw. $0,3 \mathrm{mmol} / \mathrm{l}$ zwischen den thermisch neutralen und den kühlen Arbeitsbedingungen ist jeweils leicht signifikant $(\mathrm{p} \leq 0,05)$.

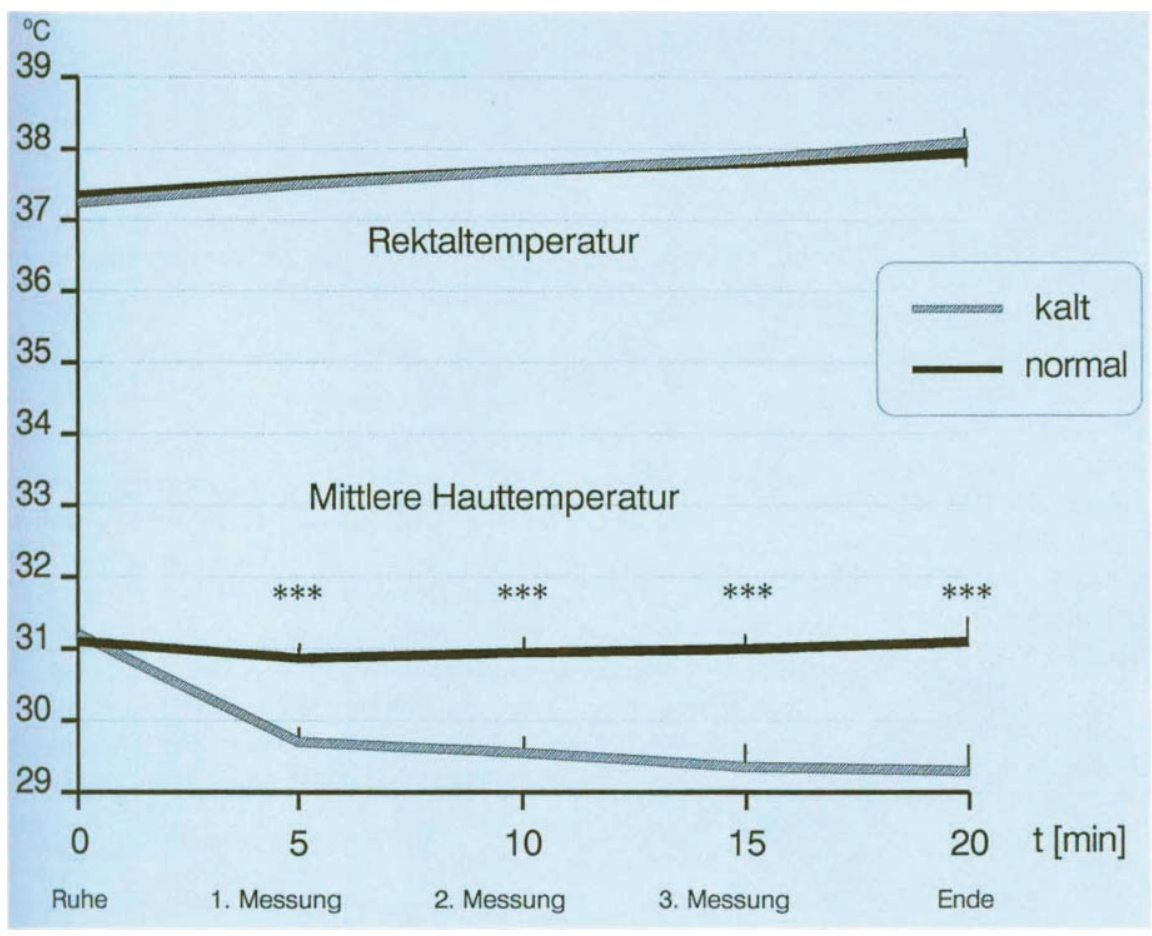

Abb. 1 Ablauf der mittleren Hauttemperatur und der Rektaltemperatur bei Begehung unter normalen und kühlen thermischen $\mathrm{Be}$ dingungen. Vorgegebene Gehgeschwindigkeit und Bekleidung $(n=32)$. Anfang, drei Meßplätze und Ende des Kurübungsweges 

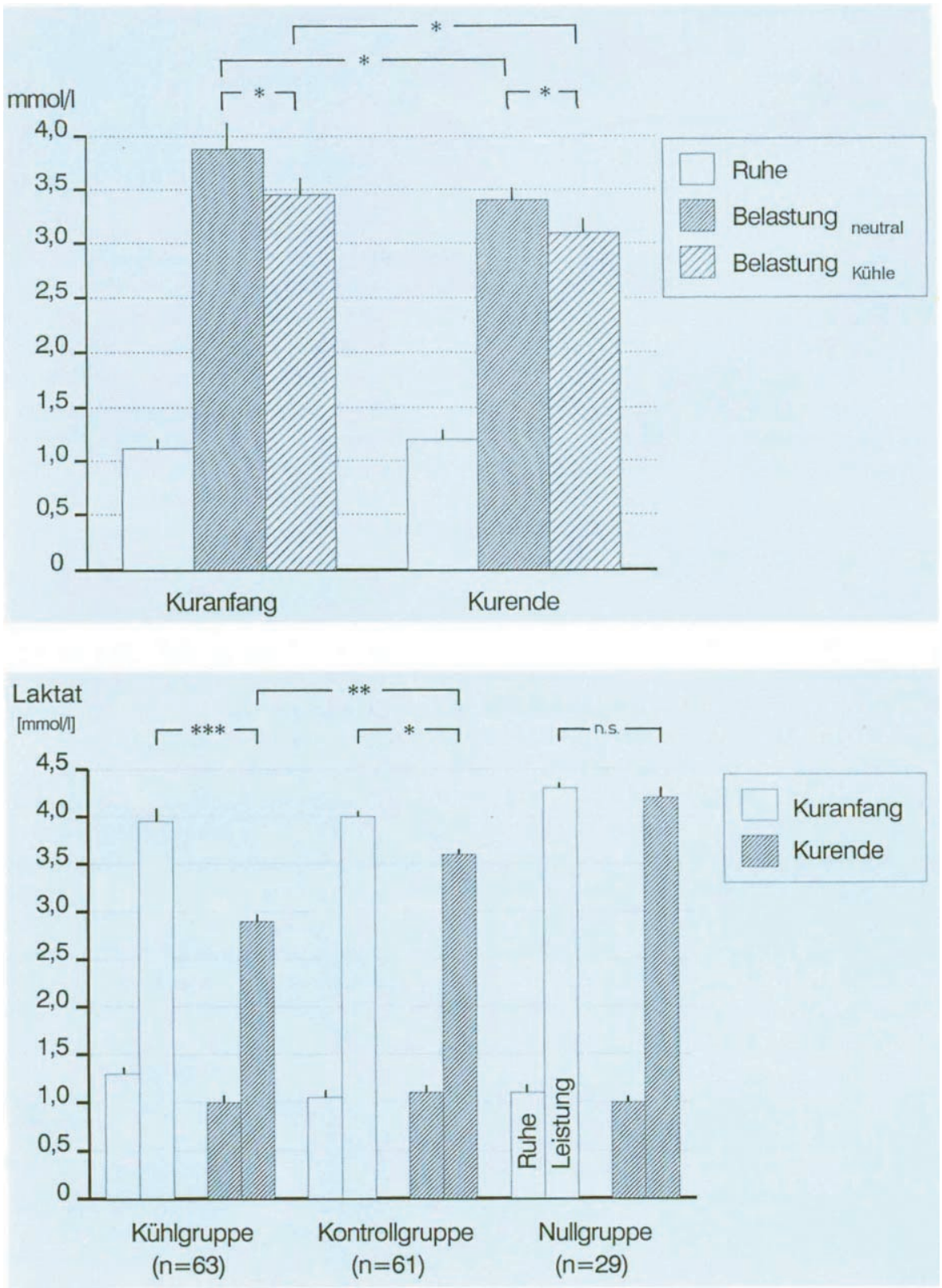

Abb. 2 Laktatspiegel unter thermisch normalen und kühlen Bedingungen. Geländetest am Kuranfang und Kurende $(n=42)$. Patienten der Kontrollgruppe. Aktuelle Leistungstähigkeit

Abb. 3 Laktatspiegel vor und nach Fahrradergometrie (thermisch normale Testbedingungen), Kuranfang und Kurende, für Kühl$(n=63)$, Kontroll- $(n=61)$ und Nullgruppe $(n=29)$. Trainingseffekt

\section{Ausdauertraining}

Nach dem 3wöchigen Training wiesen die Patienten der Kühlgruppe bei gleicher Belastung einen deutlich geringeren $(\mathrm{p} \leq 0,01)$ Laktatanstieg als die Patienten der Kontrollgruppen auf (Abb. 3): Die Patienten der Kühlgruppe haben nach dem Fahrradergometertest am Kuranfang einen Laktatspiegel von 4,0 $\mathrm{mmol} / \mathrm{l}$, der sich am Kurende bei Belastung hochsignifikant ( $\mathrm{p} \leq$ $0,001)$ um $1,1 \mathrm{mmol} / \mathrm{l}$ auf $2,9 \mathrm{mmol} / \mathrm{l}$ verringert. Bei der Kontrollgruppe erniedrigen sich die Milchsäurewerte bei Belastung nur leicht signifikant ( $\mathrm{p} \leq 0,05$ ) um $0,4 \mathrm{mmol} / \mathrm{l}$ von anfangs $4,0 \mathrm{mmol} / \mathrm{l}$ auf 3,6 mmol/l am Kurende. Die Patienten der Nullgruppe zeigen keine Veränderungen in ihrem Milchsäurespiegel (n. s.).

Die Laktatspiegel von Kühl- und Kontrollgruppe unterscheiden sich am Kurende bei gleicher Belastung signifikant $(\mathrm{p} \leq 0,01)$ voneinander.
Dieses Ergebnis wurde im Geländetest reproduziert. Der Unterschied zwischen Kühl- und Kontrollgruppe beträgt jeweils zwischen 0,5 und $1 \mathrm{mmol} / \mathrm{l}(\mathrm{p} \leq 0,01)$.

\section{Diskussion}

Die Ergebnisse der Studie zeigen, daß unter kühlen Bedingungen die gleiche Arbeit vermehrt aerob bewältigt wird. Zur Erzielung dieses Soforteffektes ist eine Reduzierung der mittleren Hauttemperatur um $2{ }^{\circ} \mathrm{C}$ ausreichend. Nach einem Ausdauertraining mit kühler Körperschale kommt es zusätzlich zu einer kältebedingten Verstärkung des aeroben Trainingseffektes. Diese Verstärkung entspricht hinsichtlich der Größenordnung den aus der Literatur bekannten Veränderungen im Laktatspiegel, die durch ein leichtes Ausdauertraining hervorgerufen werden: Der Trainingseffekt wird unter den gewählten Trainingsbedingungen annähernd verdoppelt. 
Zur Begründung der Beeinflussung der aeroben Kapazität der Arbeitsmuskulatur durch den Soforteffekt der kühlen Bedingungen und durch die Kälteadaptation beim Training stehen drei Hypothesen zur Diskussion:

1. Während Arbeit unter kühlen Bedingungen werden verhältnismäßig mehr rote Muskelfasern aktiviert (2); im Verlauf der Kälteadaptation wird das neue Rekrutierungsverhältnis immer deutlicher bevorzugt.

Das Ergebnis der vorliegenden Studie, daß durch eine Absenkung der Körperschalentemperatur während Arbeit der aerobe Metabolismus verstärkt ist, wird durch eine weitere Untersuchung bestätigt (2): Durch Absenkung der mittleren Körpertemperatur ist die akute Leistungsfähigkeit gesteigert und eine gegebene Belastung kann länger durchgehalten werden.

Aufgrund von Literaturangaben $(10,12)$ kann man vermuten, daß im kühlen Muskel überwiegend langsame rote, $d . h$. vermehrt aerob arbeitende Muskelfasern aktiviert werden. Dank ihrer Enzymausstattung können sie die arbeitslimitierende Milchsäure abbauen. Daraus ergibt sich die Hypothese 1, daß durch Abnahme der mittleren Körpertemperatur vermehrt die langsamen, auf Ausdauer spezialisierten roten Muskelfasern eingesetzt werden, d. h. daß das nerval gesteuerte Rekrutierungsverhältnis durch die mittlere Körpertemperatur beeinflußt wird. Durch Kälteadaptation wird die vermehrte Rekrutierung der aeroben Muskelfasern beibehalten. Durch ein Training bei niedrigen mittleren Körpertemperaturen wird das Verhältnis zugunsten der roten Fasern weiter verbessert.

Die Absenkung der mittleren Körpertemperatur aufgrund der Umgebungsverhältnisse resultiert aus der Erniedrigung der mittleren Haut- und Kerntemperatur. Während des untersuchten Ausdauertrainings unter kühlen Bedingungen verändert sich die Kerntemperatur indessen nicht; es muß demnach die Kühlung der Haut allein zur unterschiedlichen Rekrutierung der Motoneurone und damit der motorischen Einheiten ausreichen.

2. Das Training unter kühlen Bedingungen führt zu einer besonders ausgeprägten Muskel-Kapillarisierung, die ihrerseits den entscheidenden Parameter für die Verbesserung der aeroben Leistungsfähigkeit darstellt.

Während eines Ausdauertrainings vergrößert sich die Kapillaroberfläche der trainierten Muskulatur. Dadurch kommt es unter Arbeit zu einer Zunahme des Stromvolumens im Muskel und gleichzeitig zu einer Verkürzung der Diffusionsstrecke zwischen Kapillaren und Mitochondrien; die Diffusion des Sauerstoffs von den Kapillaren zu den Mitochondrien wird erleichtert (5).

Bei Arbeit unter kühlen Bedingungen wird zusätzlich wegen der durch die arterielle Vaso- und Venokonstriktion eingeschränkten Durchblutung der Haut das Stromvolumen verstärkt in die Muskulatur umverteilt (20): Der stärkere Flow in der Peripherie führt zur Öffnung bzw. zum Ausbau präformierter Kapillaren oder zu deren Neubildung, das $\mathrm{O}_{2}$-Angebot an die Mitochondrien ist von Anfang an vergrößert. Studien von Hollmann et al. (6) geben Hinweise darauf, daß die Mitochondrien - entgegen der bisherigen Kenntnisse - das vermehrte $\mathrm{O}_{2}$-Angebot ausschöpfen können: Nach sechswöchigem Ergometertraining unter $\mathrm{O}_{2}$-Atmung war die aerobe Energiebereitstellung vergrößert; die Laktatbildung war gegenüber der Kontrolle (Luftatmung) erniedrigt.
Bei wiederholter Arbeit - einem Training - unter kühlen Bedingungen ist so ein Anreiz zu einer adaptativen VergröBerung der Kapillaroberfläche gegeben. Durch verstärkte Vasokonstriktion der Haut als Zeichen einer Kälteadaptation $(1,3)$ kann dieser Effekt zusätzlich verstärkt werden. Als Resultat ergibt sich eine Vergrößerung der aeroben Kapazität der Energiebereitstellung und damit ein verringerter Laktatspiegel.

\section{Unter kühlen Arbeitsbedingungen wird der} Fettstoffwechsel vermehrt aktiviert.

Mehrere Autoren beschrieben eine vermehrte Aktivierung des Fettstoffwechsels bei Arbeit unter Kälte auf Kosten des anaerob-laktaziden Metabolismus. Bei Arbeit unter Gesichtskühlung wurde ein Anstieg des Fettstoffwechsels gefunden (18, 19). Eine andere Arbeitsgruppe (9) beschreibt einen intensivierten Fettstoffwechsel bei Arbeit unter kühlen Bedingungen; die Autoren sehen eine Senkung der mittleren Haut- und Kerntemperatur als ursächlich an.

Auf eine Verstärkung des Fettstoffwechsels bei Training unter Kälte weisen auch Untersuchungen an kanadischen Soldaten (14) hin: Die Probanden zeigten unter arktischen Bedingungen innerhalb von l-2 Wochen einen deutlichen Verlust an Unterhautfettgewebe. Die Autoren wiederholten den Versuch in der Klimakammer im Crossover unter kalten und normalen thermischen Bedingungen. Da eine signifikante Korrelation zwischen der Abnahme der Hauttemperatur und dem Fettverlust bestand, scheint der Hauptstimulus zur Fettstoffwechselmobilisation die lokale Kühlung der Hautoberfläche darzustellen.

Kühle Bedingungen und gleichzeitiges Training können sich in ihrem Effekt der vermehrten Fettstoffwechselaktivierung addieren. Bis jetzt existieren jedoch nur indirekte Hinweise auf eine Adaptation bei großer Kälte. Eine Untersuchung der Fettstoffwechselparameter bei einem Teil der Probanden (24) unterstützt ebenfalls Hypothese 3 .

Die drei Hypothesen sollen Gegenstand weiterführender Untersuchungen sein.

\section{Schlußfolgerung}

Die Vergrößerung der aeroben Kapazität des Muskelstoffwechsels aufgrund der kühlen Körperschale erleichtert die aktuelle aerobe Bewältigung der Arbeit und verdoppelt den Ausdauertrainingseffekt. Damit haben diese Ergebnisse eine klinische Relevanz für Patienten, welche nur leicht belastbar sind: Adaptationen werden schon bei relativ geringer Trainingsintensität ermöglicht. Dadurch wird der Ganzkörpertrainingsmangel von Kurpatienten mit Funktionsstörungen des Herz-Kreislauf-Systems ohne Organbefund stärker reduziert als aufgrund eines konventionellen Ausdauertrainings.

\section{Literatur}

1 Brück, $K$.: Basic mechanisms in thermal long-term and short-term adaptation. J. Therm. Biol. II (1986) 73-77

2 Brück, K.: Warmlaufen oder Kaltstart? Sportliche Höchstleistung durch Kälte. Spieg. Forsch. 5 (1987) 13-16

3 Brück, K.: Physiologische Grundlagen der Kälteabwehrreaktion des Menschen. Z. Phys. Med. Baln. Med. Klim. 17 (1988) 183-195

4 Brück, K. H. Olschewski: Body temperature related factors dimishing the drive to exercise. Can. J. Physiol. Pharmacol. 65 (1987) 1274-1280 
5 Hollmann, W., R. Rost, B. Dufaux, H. Liesen: Prävention und Rehabilitation von Herz-Kreislaufkrankheiten durch körperliches Training. 2. Auflage, Hippokrates, Stuttgart 1983

6 Hollmann, W., H. Liesen, E. Eghert, H. Heck, A. Mader, R. Rost, J. Satomi: Über den -Einfluß eines einbeinig durchgeführten Fahrradergometerausdauertrainings auf spiroergometrische Kriterien und das arterielle Laktatverhalten unter Luft- und Sauerstoffatmung. Dtsch. Zschr. f. Sportmed. 35 (1984) 134-141

7 Hoppeler, H., H. Howald, K. Conley, S. L. Lindstedt, H. Claassen, P. Vock, E. R. Weibel: Endurance training in humans: aerobic capacity and structure of skeletal muscle. J. Appl. Physiol. 59 (1985) 320-327

8 Hüllemann, K.-D.: Funktionelle Herzbeschwerden. In: Hüllemann, $K$.$D$. (Hrsg.): Präventivmedizin. Stufendiagnostik, Therapieleitlinien und Beratung. Thieme, Stuttgart, New York (1982) 76-82

9 Hurley, B. F., E.M. Haymes: The effects of rest and exercise in the cold on substrate mobilization and utilization. Aviat. Space Environ. Med. 53 (1982) 1193-1197

10 Kozlowski, S., Z. Brzezinska, B. Kruk, H. Kaciuba-Uscilko, J. E. Greenleaf, K. Nazar: Exercise hyperthermia as a factor limiting physical performance: temperature effect on muscle metabolism. J. Appl. Physiol. 59 (1985) 766-773

11 LeBlanc, J., B. Blais, B. Barabé, J. Côté: Effects of temperature and wind on facial temperature, heart rate and sensation. J. Appl. Physiol. 40 (1976) 127-131

12 LeBlanc, J., S. Dulac, J. Côté, B. Girard: Autonomic nervous system and adaptation to cold in man. J. Appl. Physiol. 39 (1975) 181-186

13 McArdle, W. D., J. R. Magel, G. R. Lesmes, G. S. Pechar: Metabolic and cardiovascular adjustment to work in air and water at 18,25 and 33 ${ }^{\circ} \mathrm{C}$. J. Appl, Physiol. 40 (1976) 85-90

14 O'Hara, W. G., C. Allen, R. G. Shepard, G. Allen: Fat losses in the cold - a controlled study. J. Appl. Physiol. Resp. Environm. Exercise Physiol. 46 (1979) 872

15 Olschewski, H., K. Brïck: Thermoregulatory, cardiovascular and muscular factors related to exercise after precooling. J. Appl. Physiol. 64 (1988) 1-9

16 Olschewski, H., A. Massias, K. Brück: Effect on precooling and pedal rate on aerobic and anaerobic metabolism ans exercise performance (Abstract Volume). Joint Meeting of the Physiological Society und der Deutschen Physiologischen Gesellschaft (65th meeting), Würzburg, 16.-19. März 1988
17

Ramanathan, N. L.: A new weight system for mean surface temperature of the human body. J. Appl. Physiol. 19 (1964) 531-533

18 Riggs, Ch. E.jr., D.J.Johnson, B.J. Konopka, R. D. Kilgour: Exercise heart rate response to facial cooling. Eur. J. Appl. Physiol. 47 (1981) 323-330

19 Riggs, C.E.jr., D.J.Johnson, R. D. Kilgour, B.J. Konopka: Metabolic effects of facial cooling in exercise. Aviat. Space Environ. Med. 54 (1983) 22-26

20 Rowell, L. B.: Cardiovascular adjustments to thermal stress. In: Shepard, J. T., F. M. Abboud (Eds.): Handbook of Physiology, Section 2: The cardiovascular system. Volume III, Chapter 27, 967-1023. Am. Physiol. Soc., Bethesda/Maryland 1983

21 Schuh, A.: Klimatische Einflüsse auf die Bewegungstherapie. Dissertation, München 1984

22 Schuh, A.: Das Krankheitsbild des Trainingsmangels und seine Behandlung durch Klimatherapie in Form einer Terrainkur unter kühlen Bedingungen. Habilitationsschrift, Medizinische Fakultät der Universität München 1989

23 Schuh, A., K. Dirnagl, M. Borgs: Training und Kälte in der Klimakur. Untersuchungen in Garmisch-Partenkirchen, Z. Phys. Med. Baln. Med. Klim. 15 (1986) 371-374

24 Schraudolf, M., A. Schuh: Lipoproteinveränderungen bei Frauen durch aerobes körperliches Training unter kühlen Bedingungen. Unveröffentlicht

25 v. Uexküll, T., K. Köhle: Funktionelle Syndrome in der inneren Medizin. In: $T$. yon Uexküll (Hrsg.): Psychosomatische Medizin. 3. Auflage (1986) 489--502. Urban \& Schwarzenberg, München, Wien, Baltimore

26 Vogelaere, P., R. Bekaert, R. Leclerq, M. Brasseur. A. Quirion: Preliminary study about cold stress work adaptability and recupation. Disch. Zschr. f. Sportmed. 35 (1984) 23

Priv.-Doz.Dr.Dr. med. habil. Angela Schuh

Institut für Medizinische Balneologie und Klimatologie der Universität München

Marchioninistraße 17

W-8000 München 70 